EPJ Web of Conferences 41, 01009 (2013)

DOI: $10.1051 /$ epjconf/20134101009

C) Owned by the authors, published by EDP Sciences, 2013

\title{
Attosecond X-ray free electron laser
}

\author{
S. Kumar ${ }^{1}$, H. S. Kang ${ }^{1}$, D. E. Kim ${ }^{2}$ \\ ${ }^{1}$ Pohang Accelerator Laboratory, San 31, Hyoja-dong, Pohang, Kyungbuk, 790-784, South Korea \\ ${ }^{2}$ Department of Physics, Center for Attosecond Science and Technology (CASTECH), Pohang \\ University of Science and technology (POSTECH), Pohang, 790-784, South Korea
}

\begin{abstract}
For a real, meaningful pump-probe experiment with attosecond temporal resolution, an isolated attosecond pulse is in demand. In this vein we report the generation of an isolated $\sim 148$ attosecond pulse duration radiation pulse at 0.1 angstrom wavelength using current enhanced self-amplified spontaneous emission free electron laser. We consider the 10-GeV PAL-XFEL e-beam for enhanced self-amplified spontaneous emission (ESASE) scheme. In ESASE, X-ray SASE FEL is combined with a femtosecond laser system. An $800 \mathrm{~nm}$ wavelength, 5 fs FWHM carrier envelope phase stabilized laser was employed in ESASE scheme.
\end{abstract}

\section{Introduction}

Current synchrotrons are limited up to picosecond time duration x-ray pulses, which are not capable to follow the electron dynamics in atoms, molecules and nanoscopic systems. These investigations demand tools with attosecond temporal and nanometer spatial resolutions.

Future hard x-ray FEL facility either have been proposed or are under construction [1-3]. The first successful single pass SASE FEL at $32 \mathrm{~nm}$ with a pulse duration of 25 femtosecond FWHM has been reported in 2006 [4]. Later the operation at $13.7 \mathrm{~nm}$ was reported [5]. In the saturation regime, the peak energy of $170 \mu \mathrm{J}$ was obtained. The average energy per pulse reached $70 \mu \mathrm{J}$. The pulse duration was in the region of 10 femtosecond, and the peak power of $10 \mathrm{GW}$ was achieved. SPring-8 Compact SASE source (SCSS) in Japan demonstrated the saturation at wavelengths ranging from 51 to $61 \mathrm{~nm}$ with a maximum pulse energy of $30 \mu \mathrm{J}$ from a $55 \mathrm{~m}$ long compact SASE source operating at a low acceleration energy of $250 \mathrm{MeV}$ [1]. In the hard x-ray region, LCLS at Stanford Linear accelerator (SLAC) is the first hard x-ray FEL machine, which is in operation since 2009. This facility has tunable radiation wavelength from 2.2 to $0.12 \mathrm{~nm}$ by varying the electron energy in the range of 3.5 to $15 \mathrm{GeV}$ [6].The interaction of carrier envelope phase (CEP) stabilized few-cycle laser with electron-bunch in a wiggler magnet with single or double period prior to x-ray undulator in SASE FEL was employed. It was noticed that the interaction plays a vital role for the generation of isolated attosecond $\mathrm{X}$-ray pulses with FEL [7-9]

\section{ESASE scheme for hard X-ray pulse generation}

We consider a simpler enhanced SASE scheme as shown in Fig. 1. An electron beam from a linear accelerator is sent to a double-period wiggler magnet. At the same time, a few-cycle CEP

This is an Open Access article distributed under the terms of the Creative Commons Attribution License 2.0, which permits unrestricted use, distribution, and reproduction in any medium, provided the original work is properly cited. 
stabilized laser co-propagates to induce the energy modulation in the electron beam inside the wiggler. Only small a longitudinal section of the electron beam interacts with the laser. The electron beam now enters into a chicane, which introduces dispersion in a chicane: the higher the electron energy is, the shorter it travels. In general, this leads to the density modulation, producing the microbunching of the electrons at a laser wavelength spacing and periodic current peaks. Finally, the electron-bunch enters into a long undulator to produce perfect microbunches and radiation at x-ray wavelength via standard SASE process.

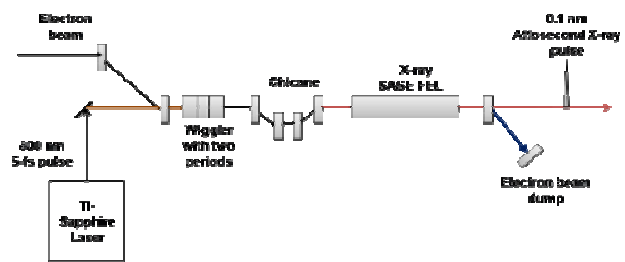

Fig. 1. ESASE scheme for attosecond pulse generation.

\section{Isolated Attosecond pulse generation}

\subsection{Generation of single current spike by energy and spatial profile of e-bunch}

We have studied the effect of laser wavelength on the current profile to obtain an attosecond hard $\mathrm{x}$-ray pulse, using electron beam parameters similar to those of the electron beam of the PAL XFEL [3]: a beam energy of $10 \mathrm{GeV}$, a total electron-bunch charge $0.2 \mathrm{nC}$, a beam normalized emittance of $0.5 \mu \mathrm{m}-\mathrm{rad}$, an electron-bunch average-current of $3 \mathrm{kA}$ or higher. A $1200 \mathrm{~nm}, \sim 13 \mathrm{GW}$ laser, pulse duration 7.5 femtosecond FWHM is used to modulate the electron beam energy. The laser is CEP stabilized. The beam waist at focus is $250 \mu \mathrm{m}$. The wiggler magnet is a two-period magnet with $\mathrm{B}_{0}=$ 1.1459 Tesla and $\lambda_{\mathrm{w}}=55 \mathrm{~cm}$. The electron beam properties are calculated by using the sixdimensional particle tracking code, ELEGANT [10].

Three different energy distributions of electron-bunch are studied which are as follows: $(1) \sim 20$ $\mu \mathrm{m}$ ( $\sim 66 \mathrm{fs}$ ) long electron-bunch with an average current of $3 \mathrm{kA}$ (Fig. 2 (a)), (2) 16 $\mu \mathrm{m}(\sim 53 \mathrm{fs})$ long electron-bunch with an average current of 4 kA (Fig. 2 (b)) and (3) $\sim 12 \mu \mathrm{m}(\sim 40 \mathrm{fs})$ long electron-bunch with an average current $6 \mathrm{kA}$ (Fig. 2 (c)). These electron-bunch distributions are generated by using ELEGANT code. The momentum compaction factor $\mathrm{R}_{56}$ is chosen between the good quality of electron beam and the size of chicane. These three electron-beam energy distributions are utilized for ESASE scheme.
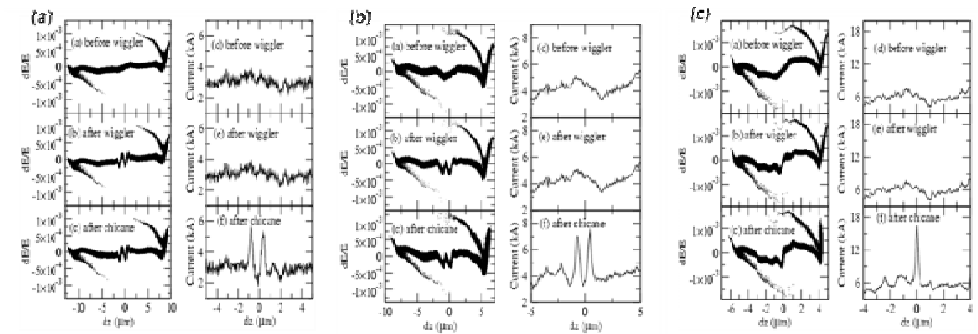

Fig. 2 The longitudinal energy distribution and current profile at three different locations : before wiggler, after wiggler and after chicane. (a) $3 \mathrm{kA}, 20 \mu \mathrm{m}$ electron bunch. (b) $4 \mathrm{kA}, 16 \mu \mathrm{m}$ electron bunch. (c) $6 \mathrm{kA}, 12 \mu \mathrm{m}$ electron bunch, respectively. 


\subsection{Single isolated Attosecond radiation pulse generation}

We present $\mathrm{x}$-ray radiation for three different electron-bunch distributions discussed in the previous section. The electron bunch is sent into a $100-\mathrm{m}$ long undulator. The radiation produced by these modulated electron bunches in the undulator is computed by a three-dimensional time-dependent FEL code GENESIS [11]. A $1200 \mathrm{~nm}$ and 7.5 femtosecond FWHM laser have modified these bunches. Since the SASE radiation is based on the build-up from noise, each electron-bunch has different behaviors, different saturation points, and different oscillation pattern.

Figure 3 (a) shows the plot of the radiation power generated at $z=73$ meter for an electronbunch of $3 \mathrm{kA}$. The typical peak power is $10 \mathrm{GW}$. Figure 3 (b) shows the linear plot of the radiation power generated at $\mathrm{z}=55$ meter for an electron-bunch of $4 \mathrm{kA}$. The typical peak power is $\sim 14 \mathrm{GW}$, on average. Figure 3 (c) shows the plot of the radiation power measured at $z=34$ meter for an average current of $6 \mathrm{kA}$. For this electron beam only a 34 meter long undulator is sufficient to produce the saturated power. The pulse duration of this peak is $\sim 146$ attosecond FWHM and the typical peak power is $58 \mathrm{GW}$. The saturation position is the point where the exponential growth of the radiation power stops.
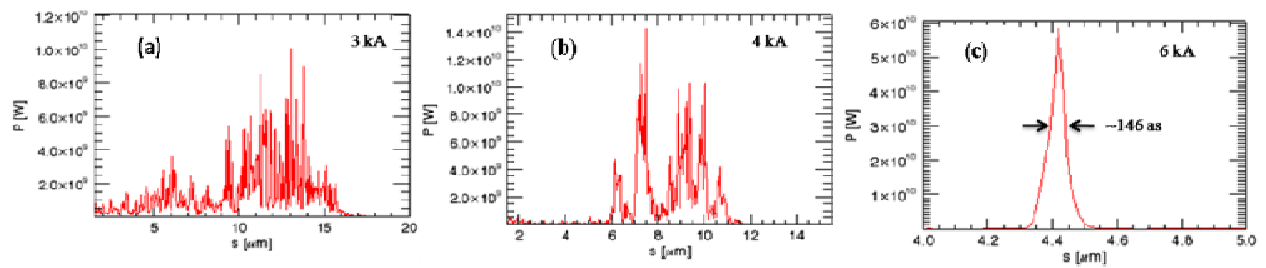

Fig. 3. (Color online) Radiation power at $0.1 \mathrm{~nm}$ wavelength for three electron-bunches

The space charge effects are severe in the low energy e-beam case. They are taken into account in the gun part of PAL-XFEL. $10 \mathrm{GeV}$ is quite high energy so that space charge effects are not so important

\section{Summary}

The optimization of laser parameters (wavelengths and pulse durations) shows that $1200 \mathrm{~nm}$ and 7.5 femtosecond FWHM and $0.2 \mathrm{~mJ}$ are optimal laser parameters for producing a single attosecond current peak with a high contrast for a given $10 \mathrm{GeV}$ electron beam of the PAL XFEL. It is demonstrated in simulation that the manipulation of electron energy distribution can produce a high contrast single peak in an electron bunch. Using the PAL XFEL parameters, we have shown that an isolated 146 attosecond, $58 \mathrm{GW}$ peak-power x-ray pulse at $0.1 \mathrm{~nm}$ is expected to be generated in a 34-meter long undulator.

\section{References}

1. T. Shintake, Nat. Photonics 2, 555 (2008).

2. B. D. Patterson, New J. Phys. 12, 035012 (2010). (see also http://fel.web.psi.ch/).

3. H. S. Kang, S. H. Nam, (Aug. 23-27, 2010). (http://fel2010.maxlab.lu.se/).

4. V. Ayvazyan, et al. Eur. Phys. J. D 37, 297 (2006).

5. W. Ackermann, Nat. Photonics 1, 336 (2007).

6. P. Emma, 4, 641 (2010).

7. A. A. Zholents, W. M. Fawley, Phys. Rev. Lett. 92, 224801 (2004).

8. A. A. Zholents, M. S. Zolotorev, New J. of Phys. 10, 025005 (2008).

9. S. Kumar, H. S. Kang, D. E. Kim, Opt. Express, 19, 7537 (2011).

10. M. Borland, http://www.aps.anl.gov

11. S. Reiche, Nucl. Instrum. Methods Phys. Res., Sect. A 429, 243, (1999). http://pbpl.hysics.ucla.edu/ reiche/ 